Georgian Mathematical Journal

$\mathbf{1}(1994)$, No. 6, 675-685

\title{
ON THE OSCILLATION OF SOLUTIONS OF FIRST ORDER DELAY DIFFERENTIAL INEQUALITIES AND EQUATIONS
}

\author{
R.KOPLATADZE AND G.KVINIKADZE
}

\begin{abstract}
Oscillation criteria generalizing a series of earlier results are established for first order linear delay differential inequalities and equations.
\end{abstract}

1. Introduction. It is a trivial consequence of the uniqueness of solutions of initial value problems that a first order linear ordinary differential equation cannot have oscillatory solutions. As to the equation

$$
u^{\prime}(t)+p(t) u(\tau(t))=0,
$$

the introduction of a delay leads to the fact that oscillatory solutions do appear. Moreover, if $p$ is nonnegative and the delay is sufficiently large, all proper solutions (see Definition 1 below) turn out to be oscillatory. Specific criteria for the oscillation of proper solutions of linear delay equations were for the first time proposed by A.D.Myshkis (see [1]). It follows from the results of $[2,3]$ that if the functions $p: R_{+} \rightarrow R_{+}\left(R_{+}=[0,+\infty[)\right.$ and $\tau: R_{+} \rightarrow R$ are continuous, $\tau$ is nondecreasing, $\tau(t) \leq t$ for $t \in R_{+}$, $\lim _{t \rightarrow+\infty} \tau(t)=+\infty$,

$$
p^{*}=\varlimsup_{t \rightarrow+\infty} \int_{\tau(t)}^{t} p(s) d s, \quad p_{*}=\varliminf_{t \rightarrow+\infty} \int_{\tau(t)}^{t} p(s) d s
$$

and

$$
\text { either } p^{*}>1 \text { or } p_{*}>\frac{1}{e} \text {, }
$$

then the inequality

$$
u^{\prime}(t) \operatorname{sign} u(t)+p(t)|u(\tau(t))| \leq 0
$$

1991 Mathematics Subject Classification. 34K15. 
is oscillatory (see Definition 3 below).

If $p_{*} \leq 1 / e$, the condition $p^{*}>1$ can be improved. For $\tau(t) \equiv t-\tau(\tau=$ const $>0)$ such an improvement was carried out successively in $[4,5,6]$ where the condition $p^{*}>1$ was replaced, respectively, by $p^{*}>1-\frac{p_{*}^{2}}{4}$, $p^{*}>1-\frac{p_{*}^{2}}{2\left(1-p_{*}\right)}$ and

$$
p^{*}>1-\frac{1-p_{*}-\sqrt{1-2 p_{*}-p_{*}^{2}}}{2} .
$$

Below we shall prove that the condition (4) remains to be sufficient for (3) to be oscillatory when $\tau: R_{+} \rightarrow R$ is an arbitrary continuous nondecreasing function.

On the other hand, in [7] the sufficient conditions for the oscillation of all proper solutions of (3) are given which involve the classes of inequalities not satisfying (2).

In the present paper, using the ideas contained in [6] and [7], we establish some criteria for the inequality (3) to be oscillatory which imply, among others, all the above mentioned results.

2. Formulation of the main results. Throughout the paper we shall assume that $p: R_{+} \rightarrow R$ is locally integrable, $\tau: R_{+} \rightarrow R$ is continuous and

$$
p(t) \geq 0, \quad \tau(t) \leq t \text { for } t \in R_{+}, \quad \lim _{t \rightarrow+\infty} \tau(t)=+\infty .
$$

Put

$$
\begin{aligned}
& \eta^{\tau}(t)=\max \{s: \tau(s) \leq t\} \text { for } t \in R_{+}, \\
& \eta_{1}^{\tau}=\eta^{\tau}, \quad \eta_{i}^{\tau}=\eta^{\tau} \circ \eta_{i-1}^{\tau}(i=2,3, \ldots) .
\end{aligned}
$$

Definition 1. Let $a \in R_{+}$. A continuous function $u:[a,+\infty[\rightarrow R$ is said to be a proper solution of the inequality (3) if it is locally absolutely continuous on $\left[\eta^{\tau}(a),+\infty\left[\right.\right.$, satisfies (3) almost everywhere in $\left[\eta^{\tau}(a),+\infty[\right.$ and

$$
\sup \{|u(s)|: t \leq s<+\infty\}>0 \text { for } t \geq a .
$$

Definition 2. A proper solution of (3) is said to be oscillatory if the set of its zeros is unbounded from above. Otherwise it is said to be nonoscillatory.

Definition 3. The inequality (3) is said to be oscillatory if any of its proper solutions is oscillatory. 
Define

$$
\begin{aligned}
\psi_{1}(t)= & 0, \quad \psi_{i}(t)=\exp \left\{\int_{\tau(t)}^{t} p(\xi) \psi_{i-1}(\xi)\right\} \\
& (i=2,3, \ldots) \text { for } t \in R_{+}, \\
\delta(t)= & \max \{\tau(s): s \in[a, t]\} \text { for } t \in R_{+} .
\end{aligned}
$$

Theorem 1. Let $k \in\{1,2, \ldots\}$ exist such that

$$
\varlimsup_{t \rightarrow+\infty} \int_{\delta(t)}^{t} p(s) \exp \left\{\int_{\delta(s)}^{\delta(t)} p(\xi) \psi_{k}(\xi) d \xi\right\} d s>1-c\left(p_{*}\right)
$$

where $\psi_{k}, \delta$ are defined by $(7),(8), p_{*}$ is defined by (1) and

$$
c\left(p_{*}\right)= \begin{cases}0 & \text { if } p_{*}>1 / e, \\ \frac{1-p_{*}-\sqrt{1-2 p_{*}-p_{*}^{2}}}{2} & \text { if } 0 \leq p_{*} \leq 1 / e .\end{cases}
$$

Then the inequality (3) is oscillatory.

Corollary $1([7])$. Let $k \in\{1,2, \ldots\}$ exist such that

$$
\varlimsup_{t \rightarrow+\infty} \int_{\delta(t)}^{t} p(s) \exp \left\{\int_{\delta(s)}^{\delta(t)} p(\xi) \psi_{k}(\xi) d \xi\right\} d s>1
$$

where $\psi_{k}$ and $\delta$ are defined by (7), (8). Then the inequality (3) is oscillatory.

Corollary 2 (see $[6]$ for $\tau(\mathbf{t}) \equiv \mathbf{t}-\tau)$. Let $p_{*} \leq 1 /$ e and

$$
\varlimsup_{t \rightarrow+\infty} \int_{\delta(t)}^{t} p(s) d s>1-c\left(p_{*}\right)
$$

where $p_{*}, \delta$ and $c\left(p_{*}\right)$ are defined respectively by (1),(8) and (10). Then the inequality (3) is oscillatory.

Corollary $3([2])$. Let

$$
\varlimsup_{t \rightarrow+\infty} \int_{\delta(t)}^{t} p(s) d s>1
$$

where $\delta$ is defined by (8). Then the inequality (3) is oscillatory. 
Theorem $2([3])$. Let $p_{*}>1 /$ e where $p_{*}$ is defined by (1). Then the inequality (3) is oscillatory.

Theorem 3. Let $p_{*} \leq 1 / e$ and

$$
\varlimsup_{t \rightarrow+\infty} \int_{\delta(t)}^{t} p(s) \exp \left\{\lambda\left(p_{*}\right) \int_{\delta(s)}^{\delta(t)} p(\xi) d \xi\right\} d s>1-c\left(p_{*}\right),
$$

where $p_{*}, \delta, c\left(p_{*}\right)$ are defined respectively by (1), (8), (10) and $\lambda\left(p_{*}\right)$ is the least root of the equation

$$
e^{p_{*} \lambda}=\lambda
$$

Then the inequality (3) is oscillatory.

3. Some auxiliary statements. In this section we establish the estimates of the quotient $|u(\tau(t))| /|u(t)|$, where $u$ is a nonoscillatory solution of (3).

Lemma 1. Let $a \in R_{+}$and $u:[a,+\infty[\rightarrow R$ be a solution of (3) satisfying

$$
u(t) \neq 0 \quad \text { for } \quad t \geq a .
$$

Then for any $i \in\{1,2, \ldots\}$

$$
|u(\tau(t))| \geq \psi_{i}(t)|u(t)| \text { for } t \geq \eta_{i}^{\tau}(a),
$$

where the functions $\eta_{i}^{\tau}$ and $\psi_{i}(i=1,2, \ldots)$ are defined respectively by (6) and (7).

Proof. Put $x(t)=|u(t)|$ for $t \geq a$. By (3) and (13) we have

$$
x^{\prime}(t) \leq-p(t) \frac{x(\tau(t))}{x(t)} x(t) \text { for } t \geq \eta^{\tau}(a),
$$

whence

$$
x(t) \geq \exp \left\{\int_{t}^{s} p(\xi) \frac{x(\tau(\xi))}{x(\xi)} d \xi\right\} x(s) \text { for } \eta^{\tau}(a) \leq t \leq s .
$$

The inequality (14) is obviously fulfilled for $i=1$. Assuming its validity for some $i=\{1,2, \ldots\}$, by (15) we obtain

$$
x(\tau(t)) \geq \exp \left\{\int_{\tau(t)}^{t} p(\xi) \psi_{i}(\xi) d \xi\right\} x(t)=\psi_{i+1}(t) x(t) \text { for } t \geq \eta_{i+1}^{\tau}(a) .
$$


Lemma 2. Let $p_{*} \leq 1 / e$, where $p_{*}$ is defined by (1). Let, moreover, $a \in R_{+}$and $u:[a,+\infty[\rightarrow R$ be a solution of (3) satisfying (13). Then for any sufficiently small $\varepsilon>0$

$$
|u(\tau(t))| \geq\left(\lambda\left(p_{*}\right)-\varepsilon\right)|u(t)| \text { for large } t
$$

where $\lambda\left(p_{*}\right)$ is the least root of the equation (12).

Proof. In view of Lemma 1 it suffices to show that there exists $k \in\{1,2, \ldots\}$ such that

$$
\varliminf_{t \rightarrow+\infty} \psi_{k}(t)>\lambda\left(p_{*}\right)-\varepsilon .
$$

By (1) $\left.\left.p_{0} \in\right] 0, p_{*}\right]$ and $t_{0} \geq a$ can by chosen such that

$$
\int_{\tau(t)}^{t} p(s) d s \geq p_{0} \text { for } t \geq t_{0}, \quad \lambda_{0}>\lambda\left(p_{*}\right)-\varepsilon,
$$

where $\lambda_{0}$ is the least root of the equation $e^{p_{0} \lambda}=\lambda$. From (7) and (18) we can easily obtain that

$$
\psi_{i}(t) \geq \alpha_{i} \quad \text { for } t \geq \eta_{i}^{\tau}\left(t_{0}\right)
$$

where $\alpha_{1}=0, \alpha_{i}=e^{p_{0} \alpha_{i-1}}(i=2,3, \ldots)$. It is not difficult to verify that the sequence $\left\{\alpha_{i}\right\}_{i=1}^{\infty}$ is increasing and bounded from above by $\lambda_{0}$. Moreover, $\lim _{t \rightarrow+\infty} \alpha_{i}=\lambda_{0}$. This fact, together with (18) and (19), shows that (17) is true.

Remark 1. The equation $u^{\prime}+p u(t-\tau)=0$, where $p>0, \tau>0$ are constants and $p \tau \leq 1 / e$ has the solution $u(t)=e^{\lambda_{0} t}$, where $\lambda_{0}$ is the greatest root of the equation $\lambda+p e^{-\lambda \tau}=0$. Since $u(t-\tau) / u(t)=e^{-\lambda_{0} \tau}=-\frac{\lambda_{0}}{p}$ and this constant is the least root of the equation $e^{(p \tau) \lambda}=\lambda$, we see that the constant $\lambda\left(p_{*}\right)$ in (16) is exact.

Lemma 3. Let $p_{*} \leq 1 / e$, where $p_{*}$ is defined by (1) and let $\tau$ be nondecreasing. Let, moreover, $a \geq 0$ and $u:[a,+\infty[\rightarrow R$ be a solution of (3) satisfying (13). Then for any sufficiently small $\varepsilon>0$

$$
|u(t)| \geq\left(c\left(p_{*}\right)-\varepsilon\right)|u(\tau(t))| \text { for large } t,
$$

where $c\left(p_{*}\right)$ is defined by $(10)$. 
Proof. If $p_{*}=0,(20)$ is obviously fulfilled. So suppose that $0<p_{*} \leq 1 / e$ and define the sequence $\left\{\beta_{i}\right\}_{i=1}^{\infty}$ as follows:

$$
\beta_{1}=\frac{1}{4} p_{*}^{2}, \quad \beta_{i}=\beta_{i-1}^{2}+p_{*} \beta_{i-1}+\frac{1}{2} p_{*}^{2} \quad(i=2,3, \ldots) .
$$

Since $\beta_{1}<c\left(p_{*}\right), \beta_{2}-\beta_{1}=\frac{1}{16} p_{*}^{4}+\frac{1}{4} p_{*}^{3}+\frac{1}{4} p_{*}^{2}>0$ and $\beta_{i}-\beta_{i-1}=$ $\left(\beta_{i-1}-\beta_{i-2}\right)\left(\beta_{i-1}+\beta_{i-2}+p_{*}\right)$, we see that the sequence $\left\{\beta_{i}\right\}_{i=1}^{\infty}$ is increasing and bounded from above by $c\left(p_{*}\right)$. Since, moreover, $\lim _{i \rightarrow \infty} \beta_{i}=c\left(p_{*}\right)$, in order to prove the lemma it suffices to show that for any $i \in\{1,2, \ldots\}$ and $\varepsilon>0$

$$
x(t) \geq\left(\beta_{i}-\varepsilon\right) x(\tau(t)) \text { for large } t,
$$

where $x(t)=|u(t)|$ for $t \geq a$.

First show that (22) is valid for $i=1$. In view of (1)

$$
\int_{\tau(t)}^{t} p(s) d s>p_{*}-\varepsilon \text { for large } t .
$$

Therefore, since $\tau$ is nondecreasing, for any sufficienty large $t$ there exists $t^{*} \in[\tau(t), t]$ such that

$$
\int_{\tau(t)}^{t^{*}} p(s) d s=\frac{1}{2}\left(p_{*}-\varepsilon\right), \int_{\tau\left(t^{*}\right)}^{\tau(t)} p(s) d s \geq \frac{1}{2}\left(p_{*}-\varepsilon\right) .
$$

By (1) and the monotonicity of $\tau$ we have

$$
\begin{gathered}
x(\tau(t)) \geq \int_{\tau(t)}^{t} p(s) x(\tau(s)) d s \geq \int_{\tau(t)}^{t} p(s)\left(\int_{\tau(s)}^{s} p(\xi) x(\tau(\xi)) d \xi\right) d s \geq \\
\geq\left(\int_{\tau(t)}^{t} p(s)\left(\int_{\tau(s)}^{\tau(t)} p(\xi) d \xi\right) d s\right) \cdot x(\tau(\tau(t))) \text { for large } t .
\end{gathered}
$$

Since by (24)

$$
\begin{aligned}
& \int_{\tau(t)}^{t} p(s)\left(\int_{\tau(s)}^{\tau(t)} p(\xi) d \xi\right) d s \geq \int_{\tau(t)}^{t^{*}} p(s)\left(\int_{\tau(s)}^{\tau(t)} p(\xi) d \xi\right) d s \geq \\
& \geq \int_{\tau(t)}^{t^{*}} p(s)\left(\int_{\tau\left(t^{*}\right)}^{\tau(t)} p(\xi) d \xi\right) d s \geq \frac{1}{4}\left(p_{*}-\varepsilon\right)^{2} \geq \beta_{1}-\frac{\varepsilon}{2 p_{*}},
\end{aligned}
$$


$\tau(t) \rightarrow+\infty$ as $t \rightarrow+\infty, \tau$ is continuous and $\varepsilon>0$ is arbitrary, the validity of (22) for $i=1$ follows from (25).

Suppose now that (22) is true for some $i \in\{1,2, \ldots\}$. By (23) for any sufficienty large $t$ there exists $t^{*}>t$ such that

$$
\int_{\tau\left(t^{*}\right)}^{t^{*}} p(s) d s>p_{*}-\varepsilon, \quad \int_{t}^{t^{*}} p(s) d s=p_{*}-\varepsilon,
$$

which implies that $\tau\left(t^{*}\right)<t$.

Integrating (3) from $t$ to $t^{*}$ we obtain

$$
x(t) \geq x\left(t^{*}\right)+\int_{t}^{t^{*}} p(s) x(\tau(s)) d s .
$$

Since $\tau(t) \leq \tau(s) \leq \tau\left(t^{*}\right)<t$ for $s \in\left[t, t^{*}\right]$, again integrating (3) from $\tau(s)$ to $t$ and using (22),(27) and the fact that $x$ is nonincreasing, we obtain for large $t$

$$
\begin{gathered}
x(\tau(s)) \geq x(t)+\int_{\tau(s)}^{t} p(\xi) x(\tau(\xi)) d \xi \geq\left(\beta_{i}-\varepsilon\right) x(\tau(t))+ \\
+x(\tau(t)) \int_{\tau(s)}^{t} p(\xi) d \xi=x(\tau(t))\left(\beta_{i}-\varepsilon+\int_{\tau(s)}^{s} p(\xi) d \xi-\int_{t}^{s} p(\xi) d \xi\right) \geq \\
\geq\left(\beta_{i}+p_{*}-2 \varepsilon-\int_{t}^{s} p(\xi) d \xi\right) x(\tau(t)) .
\end{gathered}
$$

Substituting this into (28), taking into account that by (22) $x\left(t^{*}\right) \geq$ $\left(\beta_{i}-\varepsilon\right) x\left(\tau\left(t^{*}\right)\right) \geq\left(\beta_{i}-\varepsilon\right) x(t) \geq\left(\beta_{i}-\varepsilon\right)^{2} x(\tau(t))$ and using (27) we find

$$
\begin{gathered}
x(t) \geq x\left(t^{*}\right)+x(\tau(t)) \int_{t}^{t^{*}} p(s)\left(\beta_{i}+p_{*}-2 \varepsilon-\int_{t}^{s} p(\xi) d \xi\right) d s= \\
=x\left(t^{*}\right)+x(\tau(t))\left(\left(p_{*}-\varepsilon\right)\left(\beta_{i}+p_{*}-2 \varepsilon\right)-\right. \\
\left.\quad-\int_{t}^{t^{*}}\left(\int_{t}^{s} p(\xi) d \xi\right) d s\left(\int_{t}^{s} p(\xi) d \xi\right)\right) \geq \\
\geq\left[\left(\beta_{i}-\varepsilon\right)^{2}+\left(p_{*}-\varepsilon\right)\left(\beta_{i}+p_{*}-2 \varepsilon\right)-\frac{1}{2}\left(p_{*}-\varepsilon\right)^{2}\right] x(\tau(t)) .
\end{gathered}
$$


Since $\varepsilon>0$ is arbitrary, by (21) this completes the proof of the induction step.

4. Proofs of the theorems. Proof of Theorem 1. Suppose, to the contrary, that the inequality (3) has a nonoscillatory solution $u:[a,+\infty[\rightarrow R$ and put $x(t)=|u(t)|$ for $t \geq a$. As seen while proving Lemma 1 , the inequality (15) holds. So, according to this lemma,

$$
x(\delta(s)) \geq \exp \left\{\int_{\delta(s)}^{\delta(t)} p(\xi) \psi_{k}(\xi) d \xi\right\} x(\delta(t)) \text { for } \eta_{k+1}^{\tau}(a) \leq \delta(t) \leq s \leq t .
$$

Substituting this into (3) and integrating with respect to $s$ from $\delta(t)$ to $t$, we obtain

$$
x(t)-x(\delta(t))+x(\delta(t)) \int_{\delta(t)}^{t} p(s) \exp \left\{\int_{\delta(s)}^{\delta(t)} p(\xi) \psi_{k}(\xi) d \xi\right\} d s \leq 0 .
$$

Since by Lemma 3 (20) is fulfilled for any $\varepsilon>0$ the last inequality implies

$$
\int_{\delta(t)}^{t} p(s) \exp \left\{\int_{\delta(s)}^{\delta(t)} p(\xi) \psi_{k}(\xi) d \xi\right\} d s \leq 1-c\left(p_{*}\right)+\varepsilon
$$

for large $t$, which contradicts (9).

Proof of Theorem 2. Note that the condition $p_{*}>1 / e$ implies

$$
\varlimsup_{t \rightarrow+\infty} \int_{\delta(t)}^{t} p(s) d s>\frac{1}{e} .
$$

Indeed, if this is not so, then there exist $\varepsilon>0$ and a sequence $\left\{t_{i}\right\}_{i=1}^{\infty}$ such that $t_{i} \rightarrow+\infty$ as $i \rightarrow \infty$ and

$$
\int_{\delta\left(t_{i}\right)}^{t_{i}} p(s) d s \leq \frac{1}{e}+\varepsilon
$$

Putting $\widetilde{t}_{i}=\min \left\{t \in\left[0, t_{i}\right]: \tau(t)=\delta\left(t_{i}\right)\right\}$ and recalling $\lim _{t \rightarrow+\infty} \tau(t)=$ $+\infty$, we see that $\widetilde{t}_{i} \rightarrow+\infty$ as $i \rightarrow \infty$ and

$$
\int_{\tau\left(\widetilde{t_{i}}\right)}^{\widetilde{t_{i}}} p(s) d s \leq \int_{\delta\left(\widetilde{t_{i}}\right)}^{\widetilde{t_{i}}} p(s) d s \leq \frac{1}{e}+\varepsilon,
$$


which contradicts the condition $p_{*}>1 / e$. Therefore (29) is proved. By (29) there exist $t_{0} \in R_{+}$and a number $c>1 / e$ such that

$$
\int_{\delta(t)}^{t} p(s) d s \geq c \text { for } t \geq t_{0}
$$

Repeating the arguments used in proving the inequality (26), we see from (30) that

$$
\int_{\delta(t)}^{t} p(s)\left(\int_{\delta(s)}^{\delta(t)} p(\xi) d \xi\right) d s \geq \frac{c^{2}}{4} \text { for } t \geq t_{0} .
$$

On the other hand, since $\delta(t) \geq \tau(t)$ for $t \in R_{+}$and $e^{x} \geq e x$ for $x \geq 0$, by (7) and (30) we have

$$
\psi_{i}(t) \geq(e c)^{i-2} \text { for large } t(i=2,3, \ldots) .
$$

Choose a natural $k$ such that $(e c)^{k-2}>4 / e c^{2}$, i.e. $\widetilde{c}=c(e c)^{k-1} / 4>1$. Then by (31) and (32)

$$
\int_{\delta(t)}^{t} p(s) \exp \left\{\int_{\delta(s)}^{\delta(t)} p(\xi) \psi_{k}(\xi) d \xi\right\} d s \geq \widetilde{c}>1 \text { for large } t .
$$

This means that the conditions of Corollary 1 are fulfilled. Therefore the inequality (3) is oscillatory.

Proof of Theorem 3. By (11) there exists $\varepsilon \in] 0, \lambda\left(p_{*}\right)[$ such that

$$
\varlimsup_{t \rightarrow+\infty} \int_{\delta(t)}^{t} p(s) \exp \left\{\left(\lambda\left(p_{*}\right)-\varepsilon\right) \int_{\delta(s)}^{\delta(t)} p(\xi) d \xi\right\} d s>1-c\left(p_{*}\right) .
$$

It was proved in Lemma 2 that

$$
\varliminf_{t \rightarrow+\infty} \psi_{k}(t)>\lambda\left(p_{*}\right)-\varepsilon
$$

for some natural $k$. Therefore Theorem 3 is a straighforward consequence of Theorem 1.

Remark 2. Put

$$
p(t)=\left\{\begin{array}{ll}
p_{*} & \text { for } t \in[2 k, 2 k+1[ \\
p^{*} & \text { for } t \in[2 k+1,2 k+2[
\end{array} \quad(k=0,1, \ldots),\right.
$$


$\tau(t) \equiv t-1$. It can easily be verified that

$$
\begin{gathered}
\varlimsup_{t \rightarrow+\infty} \int_{t-1}^{t} p(s) \exp \left\{\int_{s-1}^{t-1} p(\xi) d \xi\right\} d s \geq \\
\geq \lim _{k \rightarrow \infty} \int_{2 k}^{2 k+1} p^{*} \exp \left\{\int_{s-1}^{2 k} p(\xi) d \xi\right\} d s=\frac{p^{*}\left(e^{p_{*}}-1\right)}{p_{*}}
\end{gathered}
$$

Since $\left(e^{x}-1\right) / x=1+x / 2+x^{2} / 6+\circ\left(x^{2}\right)$ and

$$
\left(1-\frac{1-x-\sqrt{1-2 x-x^{2}}}{2}\right)^{-1}=1+\frac{3}{4} x^{2}+\circ\left(x^{2}\right)
$$

as $x \rightarrow 0$, we can choose $\left.p_{*} \in\right] 0,1 / e\left[\right.$ and $\left.p^{*} \in\right] p_{*}, 1[$ such that the conditions of Corollary 1 would be fulfilled for $k=2$, while those of Corollary 2 would be violated.

Consider, in conclusion, the equation

$$
u^{\prime}(t)+f\left(t, u\left(\tau_{1}(t)\right), \ldots, u\left(\tau_{m}(t)\right)\right)=0,
$$

where $m \in\{1,2, \ldots\}, f: R_{+} \times R^{m} \rightarrow R$ satisfies the local Carathéodory conditions, the functions $\tau_{i}: R_{+} \rightarrow R$ are continuous, and

$$
\tau_{i}(t) \leq t \text { for } t \in R_{+}, \quad \lim _{t \rightarrow+\infty} \tau_{i}(t)=+\infty \quad(i=1, \ldots, m) .
$$

Put

$$
\begin{aligned}
\tau(t) & =\min \left\{\tau_{1}(t), \ldots, \tau_{m}(t)\right\} \\
\delta(t) & =\max \left\{\tau_{i}(s): i \in\{1, \ldots, m\}, \quad s \in[a, t]\right\} .
\end{aligned}
$$

Definitions 1-3 are trivially extended to the equation (33).

The above results immediately imply

Theorem 4. Let (34) be valid and

$$
\begin{aligned}
& f\left(t, x_{1}, \ldots, x_{m}\right) \operatorname{sign} x_{0} \geq p(t)\left|x_{0}\right| \\
& \text { for } t \in R_{+}, \quad\left|x_{i}\right| \geq\left|x_{0}\right|, \quad x_{i} x_{0} \geq 0 \quad(i=1, \ldots, m)
\end{aligned}
$$

where $p: R_{+} \rightarrow R_{+}$is a locally integrable function. Let, moreover, the conditions of one of Theorems 1-3 or Corollaries 1-3 be fulfilled, where the functions $\tau: R_{+} \rightarrow R$ and $\delta: R_{+} \rightarrow R$ are defined by (35). Then the equation (33) is oscillatory. 


\section{ReFEREnCES}

1. A.D. Myshkis, Linear differential equations with retarded argument. (Russian) Nauka, Moscow, 1972.

2. G.Ladas, V.Lakshmikantham and L.S.Papadakis, Oscillations of higher-order retarded differential equations generated by retarded argument. Delay and Functional Differential Equations and Their Application, Academic Press, New York, 1972, 219-231.

3. R.G.Koplatadze and T.A.Chanturia, On oscillatory and monotone solutions of first order differential equations with retarded argument. (Russian) Differentsial'nye Uravneniya 18(1982), No.8, 1463-1465.

4. L.H.Erbe and B.G.Zhang, Oscillation for first order linear differential equations with deviating arguments. Differential Integral Equations 1(1988), 305-314.

5. J.Chao, Oscillation of linear differential equations with deviating arguments. Theory Practice Math. 1(1991), 32-41.

6. J.Yu and Z.Wang, Some further results on oscillation of neutral differential equations. Bull. Austral. Math. Soc. 46(1992), 149-157.

7. R.G.Koplatadze, On zeros of first order differential equations with retarded argument. (Russian) Trudy Inst. Prikl. Mat. I.N.Vekua 14(1983), $128-134$.

(Received 1.11.1993)

Authors' address:

I. Vekua Institute of Applied Mathematics

Tbilisi State University

2, University St., Tbilisi 380043

Republic of Georgia 